\title{
AUTHOR INDEX (Vol. 6)
}

Acosta, J. P., see Lezama

Ahmadpoor, M. A., see Daeichin

Ahmadpour, M. A., see Saadatnia

Alam, N. \& Khan, N. M., Special semigroup amalgams of quasi unitary subsemigroups and of quasi normal bands

Ansari-Toroghy, H. \& Farshadifar, F., On the dual notion of prime radicals of submodules

Anu, see Sharma

Aouf, M. K., see El-Ashwash

Aouf, M. K., see Seoudy

Argyros, I. K. \& George, S., Extending the applicability of Newton's method on Riemannian manifolds with values in a cone

Argyros, I. K. \& George, S., Modification of the kantorovich-type conditions for Newton's method involving twice frechet differentiable operators

Ashraf, M. \& Siddeeque, M. A., On $(\sigma, \tau)$-n-derivations in near-rings

Askari, H., see Daeichin

Askari, H., see Saadatnia

Awasthi, M. K., see Srivastava

Babaei, E., see Zamani

Baliarsingh, P., A set of new paranormed difference sequence spaces and their matrix transformations

Ballico, E., Singular curves with line bundles $L$ defined over $\mathbb{F}_{q}$ and with $H^{0}(L)=$ $H^{1}(L)=0$

Ballico, E., The Lüroth semigroup of a plane curve $C$ over
6 (2013) 1350026

\author{
6 (2013) 1350061 \\ 6 (2013) 1350019 \\ 6 (2013) 1350054
}

6 (2013) 1350010

6 (2013) 1350024

6 (2013) 1350038

6 (2013) 1350044

6 (2013) 1350032

6 (2013) 1350041

6 (2013) 1350051

6 (2013) 1350019

6 (2013) 1350054

6 (2013) 1350058

6 (2013) 1350033

6 (2013) 1350040
6 (2013) 1350023
$\mathbb{F}_{q}$ and the primitive set of $P \in C\left(\mathbb{F}_{q}\right)$

Balu, R. \& Mathew, S., On $(n$, $m$ )-iterated function systems

Bell, H. E., see Boua

Bharathi, D. \& Munirathnam, M., Simple assosymmetric rings

Bhuniya, A. K., Structure and some characterizations of left $k$-clifford semirings

Bliem, T. \& Kousidis, S., On the law of large numbers for demazure modules of $\mathfrak{s l}_{2}$

Boonchari, D. \& Saengsura, K., Idempotent and regular cohypersubstitutions of type $\tau=(3)$

Boua, A. \& Oukhtite, L., Semiderivations satisfying certain algebraic identities on prime near-rings

Boua, A., Oukhtite, L. \& Bell, H. E., Differential identities on semigroup ideals of right near-rings

Chaparro, C., see Lezama

Chaudhari, J. N., see Gupta

Christensen, O., see Christiansen

Christiansen, L. H. \& Christensen, O., Construction of smooth compactly supported windows generating dual pairs of gabor frames

Çinar, M., see Et

Cuong, D. V., Surfaces of revolution with constant Gaussian curvature in four-space

Daeichin, M., Ahmadpoor, M. A., Askari, H. \& Yildirim, A., Rational energy balance method to nonlinear oscillators with cubic term
6 (2013) 1350037

6 (2013) 1350055

6 (2013) 1350050

6 (2013) 1350047

6 (2013) 1350056

6 (2013) 1350003

6 (2013) 1350030

6 (2013) 1350043

6 (2013) 1350050

6 (2013) 1350061

6 (2013) 1350008

6 (2013) 1350011

6 (2013) 1350011

6 (2013) 1350052

6 (2013) 1350021

6 (2013) 1350019 
Darus, M., see El-Ashwash

Denecke, K. \& Srithus, R., Proper hypersubstitutions of varieties of pseudocomplemented semilattices

Dhakne, M. B., see Kucche

Dinh, H. Q., On repeated-root constacyclic codes of length $4 p^{s}$

Dragomir, S. S., Applications of Kato's inequality to operatorvalued integrals on hilbert spaces

Du, N. H. \& Liem, N. C., Linear transformations and Floquet theorem for linear implicit dynamic equations on time scales

Ebaid, A., On a new differential transformation method for solving nonlinear differential equations

Effati, S., Saberi Nik, H. \& Shirazian, M., Analyticapproximate solution for a class of nonlinear optimal control problems by homotopy analysis method

El-Ashwash, R. M., Aouf, M. K. \& Darus, M., Differential subordination results for analytic functions

Et, M., Çinar, M. \& Karakaş, M., On pointwise $\lambda$-statistical convergence of order $\alpha$ of sequence of functions

Farshadifar, F., see AnsariToroghy

Ganesa Moorthy, C., see Ramkumar

Ganesan, C., see Roopkumar

George, S., see Argyros

George, S., see Argyros

Golchin, A., see Zare

Guo, S., A method to construct coquasitriangular hopf group algebras

Gupta, V. \& Chaudhari, J. N., Right local semirings

Ida, C., Holomorphic symplectic and poisson structures on the holomorphic cotangent bundle of a complex lie group and of a holomorphic principal bundle
6 (2013) 1350044

6 (2013) 1350001

6 (2013) 1350025

6 (2013) 1350020

6 (2013) 1350059

6 (2013) 1350004

6 (2013) 1350057

6 (2013) 1350012

6 (2013) 1350044

6 (2013) 1350052

6 (2013) 1350024

6 (2013) 1350015

6 (2013) 1350005

6 (2013) 1350026

6 (2013) 1350041

6 (2013) 1350049

6 (2013) 1350013

6 (2013) 1350008

6 (2013) 1350029
Karakas,, M., see Et

Kashkarev, I., A generalization of Adjan's theorem on embeddings of semigroups

Khan, N. M., see Alam

Kousidis, S., see Bliem

Kucche, K. D. \& Dhakne, M. B., Controllability of non-densely defined abstract mixed Volterra-Fredholm neutral functional integrodifferential equations

Kurdachenko, L. A. \& Subbotin, I. Ya., The groups whose subgroups are almost ascendant

Leeratanavalee, S., see Puninagool

Lezama, O., Acosta, J. P., Chaparro, C., Ojeda, I. \& Venegas, C., Ore and goldie theorems for skew pbw extensions

Liem, N. C., see Du

Manchanda, P., see Sharma

Manna, A., see Srivastava

Mathew, S., see Balu

Mathew, S., see Minirani

Maïga, H., Integrable functions for bernoulli measures of Rank 1 - II

Mendes-Gonçalves, S. \& Sullivan, R. P., Regular elements and Green's relations in generalized transformation semigroups

Minirani, S. \& Mathew, S., Bounds for dimension of the attractor of a scaled IFS

Mohammadzadeh, H., see Zare

Munirathnam, M., see Bharathi

Nath, P. \& Singh, D. K., On a sum form functional equation related to entropies of type $(\alpha, \beta)$

Negrin, E. R., see Roopkumar

Ojeda, I., see Lezama

Oukhtite, L., see Boua

Oukhtite, L., see Boua

Prasad, A. \& Singh, V. K., Pseudo-differential operators associated to a pair of Hankel-Clifford transformations on certain beurling type function spaces
6 (2013) 1350025

6 (2013) 1350052

6 (2013) 1350022

6 (2013) 1350010

6 (2013) 1350003

6 (2013) 1350014

6 (2013) 1350016

6 (2013) 1350061

6 (2013) 1350004

6 (2013) 1350007

6 (2013) 1350018

6 (2013) 1350055

6 (2013) 1350028

6 (2013) 1350027

6 (2013) 1350006

6 (2013) 1350028

6 (2013) 1350049

6 (2013) 1350047

6 (2013) 1350036

6 (2013) 1350005

6 (2013) 1350061

6 (2013) 1350043

6 (2013) 1350050

6 (2013) 1350039 
Puninagool, W. \& Leeratanavalee, S., Natural partial ordering on $E\left(\operatorname{Hyp}_{G}(2)\right)$

Ramkumar, S. \& Ganesa Moorthy, C., Extendability of semimetrics to compactifications

Ransingh, B., Vogan diagrams of untwisted affine KacMoody superalgebras

Rao, M. S., Annulets and $\alpha$ ideals of $C$-algebras

Rao, M. S., On annihilator ideals of $C$-algebras

Rathee, S., see Ritika

Ritika \& Rathee, S., A new iterative scheme for multivalued mappings in $\mathrm{CAT}(0)$ spaces

Roopkumar, R., Negrin, E. R. \& Ganesan, C., Fourier sine and cosine transforms on boehmian spaces

Rungrottheera, W., Parameterdependent corner operators

Saadatnia, Z., Safaie, N., Ahmadpour, M. A. \& Askari, H., Higher-order energy balance method for a series of nonlinear oscillatory systems

Saberi Nik, H., see Effati

Saengsura, K., see Boonchari

Safaie, N., see Saadatnia

Seoudy, T. M. \& Aouf, M. K., On certain subclass of $P$-valent non-Bazilevic functions defined by the DziokSrivastava operator

Sharma, R. P. \& Anu Semialgebras and their algebras of differences with partial group actions on them

6 (2013) 1350038

6 (2013) 1350060

6 (2013) 1350035

6 (2013) 1350009

6 (2013) 1350053

6 (2013) 1350053

6 (2013) 1350005

6 (2013) 1350002

6 (2013) 1350054

6 (2013) 1350012

6 (2013) 1350030

6 (2013) 1350054

6 (2013) 1350032
Rao, M. S., Normal $C$-algebras

with nonuniform multiresolution analysis on positive half line

6 (2013) 1350007

6 (2013) 1350012

Sharma, V. \& Manchanda, P., Wavelet packets associated
6 (2013) 1350051

6 (2013) 1350036

6 (2013) 1350058

6 (2013) 1350039

6 (2013) 1350001

Siddeeque, M. A., see Ashraf

Singh, D. K., see Nath

Singh, S., see Srivastava

Singh, V. K., see Prasad

Srithus, R., see Denecke

Srivastava, P. D. \& Manna, A., Some difference sequence spaces generated by de la Vallée-Poussin mean

6 (2013) 1350018

Srivastava, V. K., Awasthi, M. K., Tamsir, M. \& Singh, S., An implicit finite-difference solution to one-dimensional coupled Burgers' equations

Subbotin, I. Ya., see Kurdachenko

Sullivan, R. P., see MendesGonçalves

Tamsir, M., see Srivastava

Thoang, L. D., A characterization of co-harada ring

Venegas, C., see Lezama

Wang, Y. \& Yin, Z., Two particular eventually regular semigroups with 0 -modular or 0-distributive subsemigroup lattices

Yildirim, A., see Daeichin

Yin, Z., see Wang

Zamani, Y. \& Babaei, E., Symmetry classes of polynomials associated with the dicyclic group

Zare, A., Golchin, A. \& Mohammadzadeh, H., Strongly torsion free acts over monoids
6 (2013) 1350058

6 (2013) 1350014

6 (2013) 1350006

6 (2013) 1350058

6 (2013) 1350017

6 (2013) 1350061

6 (2013) 1350046

6 (2013) 1350019

6 (2013) 1350046

6 (2013) 1350033

6 (2013) 1350049 